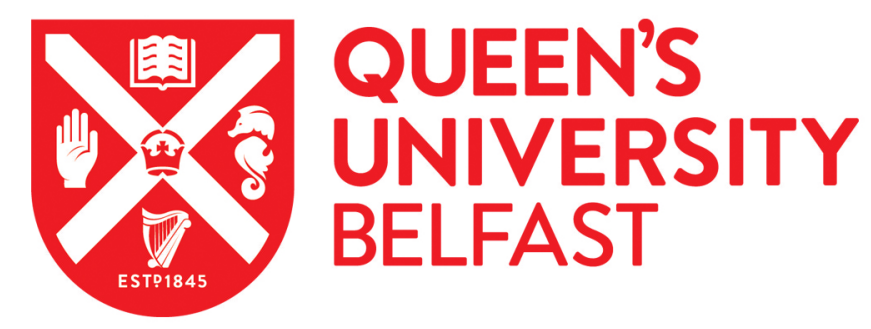

\title{
Sexes and species as rival units of niche saturation during community assembly
}

Pincheira-Donoso, D., Tregenza, T., Butlin, R., \& Hodgson, D. (2018). Sexes and species as rival units of niche saturation during community assembly. Global Ecology \& Biogeography, 27, 593-603.

https://doi.org/10.1111/geb.12722

\section{Published in:}

Global Ecology \& Biogeography

\section{Document Version:}

Peer reviewed version

Queen's University Belfast - Research Portal:

Link to publication record in Queen's University Belfast Research Portal

\section{Publisher rights}

Copyright 2018 Wiley. This work is made available online in accordance with the publisher's policies. Please refer to any applicable terms of use of the publisher.

\section{General rights}

Copyright for the publications made accessible via the Queen's University Belfast Research Portal is retained by the author(s) and / or other copyright owners and it is a condition of accessing these publications that users recognise and abide by the legal requirements associated with these rights.

Take down policy

The Research Portal is Queen's institutional repository that provides access to Queen's research output. Every effort has been made to ensure that content in the Research Portal does not infringe any person's rights, or applicable UK laws. If you discover content in the Research Portal that you believe breaches copyright or violates any law, please contact openaccess@qub.ac.uk. 
1 Sexes and species as rival units of niche saturation during community assembly

2

3 Daniel Pincheira-Donoso ${ }^{1,2,4}$, Tom Tregenza², Roger K. Butlin ${ }^{3}$ \& David J. Hodgson 2,4

4

$5{ }^{1}$ Laboratory of Evolutionary Ecology of Adaptations, School of Life Sciences, University of Lincoln, Brayford

6 Campus, Lincoln, LN6 7DL, Lincolnshire, United Kingdom

$7 \quad{ }^{2}$ Centre for Ecology and Conservation, College of Life and Environmental Sciences, University of Exeter,

8 Cornwall Campus, Penryn, TR10 9FE, Cornwall, United Kingdom

9 3Department of Animal and Plant Sciences, University of Sheffield, Western Bank, Sheffield, S10 2TN, United

10 Kingdom and Department of Marine Sciences, University of Gothenburg, 40530 Gothenburg, Sweden.

$11 \quad{ }^{4}$ Corresponding Authors: DPincheiraDonoso@lincoln.ac.uk, D.J.Hodgson@exeter.ac.uk

Key words: Adaptive radiation, speciation, divergent selection, sexual dimorphism, ecological opportunity, niche-packing, lizards, Liolaemus

18 Short Running Title: Sexes and species compete to fill niches

Words in Abstract: 299 


\section{Abstract}

31 Aim: Community assembly is traditionally assumed to result from speciation and colonisation mediated by available niche space. This paradigm is expanded by the theory that niche space can also be saturated by 7 intersexual adaptive divergence (ecological sexual dimorphism) when interspecific competition is relaxed. This theory (here termed 'niche-packing equivalence') predicts that the evolution of ecological sexual dimorphism constrains the ecological opportunity that would otherwise lead to ecological speciation or colonisation, and that saturation of niches by different species constrains divergent selection for divergence between the sexes. Therefore, sexes and species are equivalent, yet antagonistic units of niche occupation. We present the most comprehensive test of the niche-packing equivalence theory at ecological timescales (assemblage level) to date. Location: South America

Major taxa: Liolaemus lizards.

Methods: We identified 23 Liolaemus assemblages varying in species-richness and sexual size dimorphism (SSD), distributed across a wide environmental range. We used mixed effects models, permutation tests and MCMC regressions to quantify the relationship between SSD and species-richness. We then partitioned the body size niche dimension between the sexes and among species, and tested for non-overlapping body size distributions. We regressed SSD and species-richness of each assemblage against environmental predictors, using multi-model inference and structural equation modelling.

Results: Sexual dimorphism declines with increasing species-richness, and a strong signal of tension between the two remains following phylogenetic control. This pattern is accompanied by evidence of constraints on bodysize partitioning among species and between the sexes: the two units of niche saturation tend not to overlap. However, across assemblages, species-richness and SSD correlate with different environmental variables, suggesting that their tension is context-specific.

Main conclusions: Our evidence supports the prediction that sexual dimorphism and species-richness are alternative outcomes of adaptive radiation. However, this antagonism is mediated by a suite of environmental predictors that influence dimorphism and species-richness differentially. 


\section{Introduction}

The adaptive proliferation of biodiversity results from divergent natural selection driving niche expansions in species exposed to ecological opportunity - a process potentially leading to speciation (Schluter, 2000; Gavrilets, 2004; Nosil, 2012). Therefore, a prevailing paradigm in evolutionary ecology is that the distribution of biodiversity is shaped by the diversity-dependent accumulation of species that compete to saturate niche space (Losos, 2010). However, saturation of ecological opportunity by newly evolving species can be replaced by adaptive divergence between the sexes of the same species (ecological sexual dimorphism). According to this idea, intersexual niche expansions are promoted by disruptive natural selection when sexual conflict arising from resource competition is mitigated by the evolution of dimorphic males and females adapted to non-overlapping regions of the niche landscape (e.g., Fairbairn et al., 2007), in environments where the intensity of interspecific competition declines with decreasing numbers of competitors (Slatkin, 1984; Bolnick \& Doebeli, 2003).

Accumulating evidence suggests that the evolution of ecological sexual dimorphism can influence, or be influenced by the trajectories and rates of biodiversity proliferation on macroevolutionary timescales (i.e., when rates of speciation within a lineage are associated with the degree of sexual dimorphism at phylogenetic nodes) and on microevolutionary and ecological timescales (i.e., when species richness, whether resulting from speciation or colonisation, is associated with the degree of sexual dimorphism in resident species). The proliferation of sexually dimorphic species is predicted to saturate morphospace, thus increasingly limiting the opportunities for lineages to radiate adaptively via niche filling (Schoener, 1977; Losos, 2009; De Lisle \& Rowe, 2015). In any given assemblage, the saturation of niche space by an increasing number of species is expected to limit opportunities for the evolution of ecological sexual dimorphism, while niche saturation by dimorphic species might constrain colonisation by additional species (Bolnick \& Doebeli, 2003; Butler et al., 2007). Therefore, this 'niche-packing equivalence' theory predicts that ecologically distinct species and dimorphic sexes operate as rival units of niche saturation during adaptive radiations or community assembly, which leads to ecological and evolutionary tension between the two forms of diversification as each of them contributes to saturation of the ecological opportunity (Slatkin, 1984; Bolnick \& Doebeli, 2003).

The underappreciated, yet fundamental role for ecological sexual dimorphism in influencing the trajectories of lineage diversification and assemblage evolution has received limited attention that has resulted in mixed support. At macroevolutionary timescales, the only known study (De Lisle \& Rowe, 2015) presented robust evidence rejecting the core prediction that lineage diversification rates decay with increasing sexual 
dimorphism. Based on a global-scale amphibian analysis, these authors showed that increasing sexual size dimorphism (SSD) is associated with increases in speciation rates, and decreases in extinction rates. At assemblage level, a few studies have revealed conflicting evidence. On the one hand, a small number of studies on Anolis lizards (Schoener, 1969, 1977; Butler et al., 2007; Poe et al., 2007) and turtles (Stephens \& Wiens, 2009), have shown negative correlations between species-richness and sexual dimorphism, consistent with niche-packing equivalence theory. In contrast, a global-scale study investigating the effect of insularity and species-richness on the degree of sexual dimorphism across island mammals and lizards (Meiri et al., 2014) failed to identify a relationship between the two forms of diversity. Such disparate results across studies may be caused by their extreme differences in spatial and taxonomic scale. At very large scales (e.g., Meiri et al., 2014), selection may result from competition across multiple resource axes, thus potentially dissipating the predicted impetus of univariate selection operating on a specific trait that may be pushed to diverge to mitigate intersexual conflict via evolution of sexual dimorphism (Cooper et al., 2011). In addition, De Lisle \& Rowe (2015) suggested that the signal of competition is more likely to be identified at finer scales (such as in the Anolis studies), while taking into account proxies of the ecological opportunity under which diversification dynamics occur. Such proxies might include the availability of different levels of resources to accommodate ecologically different sexes or species, or the occupation of distinct portions of morphospace. No such quantitative tests of the niche-packing equivalence theory exist.

In this study, we present the most comprehensive test of the ecological-scale version of the nichepacking equivalence theory that community assembly is mediated by an antagonistic tension between the degree of sexual dimorphism and species-richness, as a function of available niche space. Using multiple assemblages of Liolaemus lizards (Pincheira-Donoso et al., 2015), one of the world's most prolific vertebrate radiations (Pincheira-Donoso et al., 2013b; Pincheira-Donoso et al., 2013a), we implemented a test that investigates the theory at a fine taxonomic scale, but at large spatial and environmental scales, and includes measures of microhabitat availability across assemblages. Following control of phylogenetic effects, we reveal the predicted negative covariation between sexual dimorphism in body size and species-richness across assemblages. We then use body size distributions per species to test whether sexes and species occupy distinct portions of the body-size phenotypic dimension. We also test whether species-richness and SSD share similar sets of environmental predictors. 


\section{Materials and methods}

\section{Assemblage selection}

We studied 23 Liolaemus assemblages consisting of one to five species (Supplementary Figure S1; Supplementary Table S1). Our assemblages occur on the western side of the Andes mountain range in Chile, where $\sim 85 \%$ of lizard species (90+ species) belong to this clade (Pincheira-Donoso et al., 2008b; PincheiraDonoso et al., 2017), and in Argentinean Patagonia, where multiple independent lizard invasions of highelevation plateaus ('Mesetas') have resulted in isolated assemblages (Cei, 1986; Scolaro, 2005; PincheiraDonoso, 2011). Boundaries of assemblages were determined by geographic (e.g., mountains, valleys, rivers) and/or ecological features (e.g., desert assemblages isolated in vegetation patches). In addition, published distributional data (e.g., Cei, 1986; Pincheira-Donoso \& Núñez, 2005) and over 8,000 museum records provided the original basis for identification of independent assemblages. Data obtained from field explorations carried out over ten years (by DP-D) provided 4,000 further geographic data points that confirmed our conclusions about assemblage isolation. Five species in our dataset were represented in a maximum of two different assemblages (see Table S1, for species names). A few other Liolaemus assemblages host 6-8 coexisting species, but given the lack of clear boundaries among these and other assemblages (e.g., substantial spatial overlap among species with large geographic ranges) they were excluded from the analyses to avoid a decay in the spatial control over species interactions and thus, of our proxy for the intensity of competition (e.g., see Butler et al., 2007; Losos, 2009). Finally, all our studied lizard assemblages are dominated by (or consist exclusively of) Liolaemus species, avoiding the competitive effects that lizards of other lineages, with potentially similar ecological requirements, might exert within each assemblage.

\section{Sexual size dimorphism data}

Body size data were collected for all species found in the 23 studied assemblages. Snout-vent length (SVL) is the standard estimator of body size in lizards (Meiri, 2008; Pincheira-Donoso et al., 2011). Hence, we used this proxy to quantify the extent of sexual size dimorphism. Given that body size in lizards follows asymptotic growth curves, the use of the largest available/known specimen or the use of the average calculated from the entire sample can overestimate or underestimate, respectively, adult body sizes (Stamps \& Andrews, 1992; Brown et al., 1999). Therefore, from the entire available sample of adult specimens (Pincheira-Donoso \& Núñez, 2005; Pincheira-Donoso \& Tregenza, 2011), we obtained the mean of the largest two-thirds of each sample per sex, 
per species, and per assemblage, which provides an intermediate SVL value (between the mean and maximum known for each sex), and hence, a more reliable estimate of adult body size (Losos et al., 2003; PincheiraDonoso et al., 2008a). Subsequently, the degree of SSD was calculated with the formula In(SVLMale/SVLFemale). This measure of dimorphism is intuitive and has been shown to perform with satisfactory statistical power (Smith, 1999; Fairbairn, 2007). The fundamental prediction of the theory is that the extent of sexual dimorphism varies as a function of assemblage species-richness. SSD estimates for the five Liolaemus species found in two assemblages (see Supplementary Table S1) were therefore calculated based on the actual specimens recorded at each specific assemblage, separately.

\section{Relationships between SSD and species-richness}

We tested for a tension between interspecific and intraspecific adaptive diversity by correlating or regressing SSD against species-richness, across our 23 assemblages. Different approaches can be taken to this analysis, each with strengths and weaknesses. The simple correlation between assemblage species-richness and SSD (the mean SSD across all species in the assemblage) is conservative, but excludes information on the individual SSD values for each species. The correlation between species-richness and individual species' SSD ignores the non-independence of species nested within assemblages. Accounting for "assemblage identity" as a random effect to avoid this pseudoreplication obliged us to use regression models that assumed no uncertainty in the predictor, species-richness. We present per-assemblage, per-species, and mixed-effects versions of these analyses, and control for artefactual relationships by permuting (shuffling) species randomly among assemblages. For each analysis, the slope or correlation coefficient of the observed relationship was compared to the null distribution of slopes based on $10 \mathrm{~K}$ permutations, concluding statistical significance if the observed correlation parameter lies in the upper or lower $2.5^{\text {th }}$ centiles of the null distribution. These analyses were adjusted for data quality and precision by weighting them by the sample sizes used to calculate sexual dimorphism for each species.

\section{Controlling for phylogenetic non-independence}

172 Recognising that observed correlations between SSD and species-richness could be due to phylogenetic 173 patterns of SSD coupled with phylogenetically biased co-occurrences of species in assemblages, we repeated our per-species regression analyses using phylogenetic control on the residuals. There exists a well-developed 
phylogeny for many Liolaemus species (Pincheira-Donoso et al., 2013a; Pincheira-Donoso et al., 2015), but only half of the species in this study are represented as tips. We created a proxy phylogeny by associating unrepresented species with sister species or closest relatives that appear in the established phylogeny. This proxy phylogeny (Supplementary Figure S2; Supplementary Table S3) contains tips that can each represent multiple "real" species in our dataset. This required us to account for the influence of phylogeny using a Monte Carlo Markov Chain (MCMC) generalised linear mixed effect regression model, which considered species identity, assemblage identity and proxy phylogeny as random effects. We used the MCMCgImm package in R, and employed parameter expansion of our three random effects to ensure convergence. MCMC chains were run for $100 \mathrm{~K}$ iterations with a burn-in of $10 \mathrm{~K}$ and a thinning interval of 100 . We report the posterior distributions of variance absorbed by phylogeny, and slope of the relationship between sexual dimorphism and speciesrichness.

\section{Relationships between body size distributions and species-richness}

We explicitly tested our assumption that species in species-poor assemblages occupy larger niches than species in species-rich assemblages by examining the predicted negative correlation between species-richness and the breadth of their body size distributions (measured as the standard deviation of SVL). As with analyses of SSD above, we tested this correlation per-assemblage, using the mean of the body size standard deviations across species. We then modelled the slope of the relationship between per-species body size standard deviation and species-richness, absorbing assemblage as a random effect. All analyses were partnered with permutation tests that shuffled species among assemblages $10 \mathrm{~K}$ times to create a histogram of test statistics under the null hypothesis of no correlation/relationship. Recognising that dimorphic species are predisposed to having broader body-size distributions, we repeated these tests separately for males and for females. This provides a check that changes in body-size distributions are due to sexual dimorphism, not expansion of each sex's size distribution.

\section{Partitioning body size variation between sexes and species}

The hypothesis that correlations between SSD and species-richness are driven by constraints on body size distributions (driven by size-dependent competition) predicts that, within assemblages, there should be a negative relationship between the proportion of variance in body size explained by partitioning among species and that explained by partitioning between the sexes. We expect a negative relationship between these 
variance components by default (because where more variance is explained by one component, less is available to be explained by the other). However, residual variance, which describes the overlap in body size distributions between sexes and among species, also contributes to total variation. If the tension between SSD and speciesrichness forces those ecological units into distinct portions of the body size niche dimension, then natural assemblages should lie closer to the line of perfect negative covariance than artificial assemblages made by shuffling combinations of species.

To test this prediction we performed a factorial analysis of variance in body size against sex (male or female) and species identity (one to five species), for each assemblage. We recorded the proportions of variance $\left(R^{2}\right)$ explained by sexes and species, and the proportion left unexplained, then modelled the nonlinear relationship between $R^{2}$ sexes and $R^{2}$ species. Because $R^{2}$ sexes $+R^{2}$ species cannot be greater than 1 , this relationship is constrained to lie below the hypotenuse between $[0,1]$ and $[1,0]$. Assemblages lying along the line are those in which all of the variation in body size is explained by a combination of sex- and species-identity effects.

Assemblages lying closer to $[0,0]$ are those in which body size distributions overlap considerably between sexes and among species (Figure 3). The null hypothesis for this analysis is that the natural assemblages of Liolaemus lie no closer to the $R^{2}$ sexes $+R^{2}$ species $=1$ hypotenuse than random assemblages of lizard species. We tested departure from this expectation by shuffling species among assemblages $10 \mathrm{~K}$ times and repeating the factorial ANOVA analyses for each shuffle. This approach combines the influence on niche saturation of divergence among species, and divergence between sexes. To test the influence of species divergence alone, we shuffled the size-differences among species, but constrained the size-differences between sexes to be as observed in the data. To test the influence of sexual divergence alone, we shuffled the size-differences between sexes, among species, but constrained the size-differences among species to be as observed in the data. Simulations of simplified assemblages occupying body size niches according to four simple rule-sets (a) random assembly of species and sexes; b) species occupy available niches preferentially, but sexual divergence is random; c) species occupy niches randomly, but sexual divergence occurs when niches are available; d) species and sexes both diverge into available niches) confirmed that these constrained shuffles correctly revealed niche-packing patterns due to sexual or species divergence (see supplementary material).

For the observed data and each shuffle (total shuffle; species shuffle; sex shuffle), we modelled the distance of $R^{2}$ sexes vs. $R^{2}$ species from the hypotenuse, in two ways. First, we calculated the mean deviation of perpendicular residuals from the hypotenuse. Second, recognising that shuffled assemblages with overlapping 
233 body size distributions lay closer to $[0,0]$ than observed assemblages, and that data close to $[0,0]$ naturally lay 234 furthest from the hypotenuse, we used least-squares nonlinear regression to test the curvature of the quadratic 235 fit to observed or simulated variance components that joined the constrained intercepts of $[0,1]$ and $[1,0]$. The quadratic function that links $x$ (the distance along the hypotenuse) to $y$ (the perpendicular distance of $\left[R^{2}\right.$ species,

$237 R^{2}$ sexes] from $\left.x\right)$, is $y=b\left(\sqrt{2} x^{2}-2 x\right)$, where $b$ describes the intensity of curvature (see Figure 3 ). Both sets of 238 analyses weighted the contribution of real and shuffled assemblages by the residual degrees of freedom of the associated ANOVA used to calculate $R^{2}$ sexes and $R^{2}$ species. We compared the observed outcome (mean deviation

240 from the hypotenuse; curvature of the quadratic) to the empirical null distributions of these parameters based on 241 our shuffles, and calculated p-values based on the quantile position of the observed parameters (Figure 3).

\section{Sexual dimorphism: sexually or naturally selected?}

244 Although not essential to the expanded niche-packing equivalence theory, we note that if SSD is driven by 245 ecological opportunity alone, there should be no trend for dimorphism to be consistently male- or female-biased. 246 Alternatively, if SSD is driven by sexual selection, we might expect males to be consistently larger than females, 247 or vice versa. We tested this with a simple paired $t$-test of mean body size between males and females, across species. We checked the robustness of this result to phylogenetic control, by fitting an intercept-only MCMCgImm, with SSD as response variable, using the proxy phylogeny, and all MCMC settings as described above.

\section{Environmental estimators of niche space abundance}

Different environments are expected to provide different diversities of potential niches to be constructed or exploited (Peterson et al., 2011). Since the assemblages we sampled are widely spread along a $\sim 3800 \mathrm{~km}$ latitudinal range, the availability of niche space is likely to vary across these assemblages, creating variation in their potential to host different numbers of ecological units, whether different species or divergent sexes within species. To examine this variation, we regressed SSD and species-richness against a number of environmental factors as proxies for niche diversity per assemblage. First, exclusively based on field observations, we quantified the numbers of microhabitats and the amount of vegetation available per assemblage site. Six microhabitat categories were identified in the areas occupied by Liolaemus (boulders, rocky ground, open ground, bushy ground, grassland, and trees) (Schulte et al., 2004; Pincheira-Donoso et al., 2009), which were 
each scored as rare (0), relatively common (0.5) or common (1). We summed these scores across microhabitat categories to yield a "microhabitats" index ranging from zero to six. Vegetation indices ranged from zero (little or no vegetation) to three (high cover of thick scrub) with intervals of 0.5 . We then employed two proxies of resource abundance (Costa et al., 2007; Pincheira-Donoso \& Meiri, 2013; Novosolov et al., 2016): mean annual precipitation (on a spatial resolution of $1 / 6^{\circ}$ ), assumed to be positively associated with productivity in the areas we study; and NPP, an estimate of the net amount of solar energy converted to plant organic matter through photosynthesis, measured in units of elemental carbon per year, on a spatial resolution of $1 / 4^{\circ}$. Precipitation data came from Worldclim (Hijmans et al., 2005) and NPP data (log-transformed) came from Imhoff et al (2004). These climatic data were assigned to each studied community by intersecting the geographical centroids of the assemblages with the above climatic layers in ArcGIS 9.3.1. To quantify the influence of environmental factors on SSD and species-richness, we performed multiple regression analysis of mean responses per assemblage against NPP, annual precipitation, latitude, altitude, vegetation and microhabitat diversity, all scaled to have zero mean and unit variance. We used Akaike Information Criteria and Akaike model weights, and dredged the full model to determine the best model and the difference in AIC for each possible subset model using the R package 'MuMIn' (Barton, 2017). This full set of models was averaged, with parameters weighted by Akaike model weights, to provide means and $95 \%$ confidence intervals for the model-averaged effect sizes of each predictor. Phylogenetic control is not applicable to these analyses because we use assemblage-level, rather than species-level metrics. We then used Structural Equation Modelling (SEM), using the R package 'sem' (Fox et al., 2017), to tease apart the relationship between environment, SSD and species richness. We used the subset of environmental predictors, identified by our multiple regressions as having significant influence on the response variables. We treated these as predictors, and considered three SEMs: first, a model in which environmental variables predicted SSD and species richness independently, but with residual covariance between these two responses; second, environmental variables predicted SSD which in turn predicted species richness; third, environmental variables predicted species richness which in turn predicted SSD. We used a combination of significance tests and AIC to compete these models, statistically.

\section{Results}

Relationship between sexual size dimorphism and species-richness 
290 As predicted, the magnitude of SSD correlated negatively with the number of Liolaemus species per assemblage

291 (Figure 1). As numbers of coexisting species per assemblage increased, there was a significant decrease in the 292 average degree of SSD per assemblage (Pearson's correlation; $\rho=-0.430, t_{21}=-3.226, P=0.004$; permutation 293 test $P$-value 0.003 ; Figure 1a). Correlation analysis using each species confirmed this result $\left(\rho=-0.387, t_{53}=-\right.$ 294 3.057, $P=0.003$; permutation $P=0.002$ ), as did mixed effects regression of SSD against species-richness, 295 weighted by sample size for each species (slope $=-0.43, F_{1,21}=12.03, P=0.002$; permutation $P=0.001$; Figure 296 1b). These patterns were robust $(P$ remained $<0.05)$ to the removal of an influential single-species assemblage 297 with high SSD (the Arica assemblage, Figure 1a, b). Indeed, $P$-values were $<0.1$ for analyses that completely 298 removed all single-species assemblages (although it would be difficult to justify such extreme data pruning). 299 Phylogenetic mixed-effects regression revealed credible phylogenetic signal in the residuals of this model 300 (Figure 1c), but the posterior distribution of the slope of sexual dimorphism against species-richness was 301 negative with $96.3 \%$ probability (Figure 1d). The variances due to assemblage and species identities were not 302 credibly greater than zero.

\section{Correlation between breadth of body size distribution and species-richness}

305 The negative correlation between SSD and species-richness was accompanied by a significant negative 306 correlation between the average breadths of body size distributions per species, and species-richness per 307 assemblage (Pearson's correlation; $\rho=-0.432, t_{21}=-2.198, P=0.039$; permutation test $P$-value 0.019; Figure 308 2a). This relationship held when the correlation was tested using each species in each assemblage, and when 309 modelled as a regression with a random effect of assemblage identity $\left(\rho=-0.323, t_{53}=-2.483, P=0.016\right.$; 310 permutation $P=0.004$; slope $=-0.430, F_{1,21}=6.167, P=0.022$; permutation $P=0.006$ ) (Figure $2 b$ ). However, no 311 significant relationship existed between the breadths of body size distributions per sex and species-richness (per 312 assemblage correlations: male body size $\rho=0.215, t_{21}=1.012, P=0.323$, Figure 2 c; female body size $\rho=$ $3130.191, t_{21}=0.891, P=0.383$, Figure $2 \mathrm{~d}$ ). All of these results were supported by MCMCgImm models that 314 controlled for phylogenetic signal. Indeed, we found no credible evidence for phylogenetic signal in the breadth 315 of body size distributions per species, nor per sex per species. 
318 The proportion of variance in body size explained by intersexual divergence decreased as the proportion 319 explained by interspecific divergence increased (Figure 3a). More importantly, we found support for the 320 prediction that this relationship is more intense (i.e., the observations lay closer to the line of perfect constraint; 321 Figure $3 b$ ) than for the vast majority of shuffled lizard assemblages created to define the expectation under the null hypothesis (see results in Supplementary Analysis S2, and Table S2). The mean deviation of the observed partition of body size variation from the line of perfect constraint was too small to fit the null hypothesis distribution (permutation $P<0.001$; Figure 3c). Constrained shuffles revealed that niche-packing as measured by this deviation was due to a combination of species divergence $(P=0.014)$ and sexual divergence $(P=0.001)$. curvature in the quadratic line joining the intercepts of "all intersexual variation" and "all interspecific variation" (Figure $3 b$ ) was too small to fit the null hypothesis (permutation $P=0.001$; Figure $3 \mathrm{~d}$ ). Constrained shuffles revealed that niche-packing, as measured by curvature, was due to a combination of species divergence $(P=$ $0.049)$ and sexual divergence $(P=0.01)$. This provides clear evidence that natural Liolaemus assemblages are structured such that the negative association between intersexual and interspecific body size variation is closer to the perfect constraint than expected by chance. Sexes and species both tend to occupy distinct portions of the body size niche dimension when niche opportunities exist, and appear to constrain each other's divergence or

\section{Is SSD generally naturally or sexually selected?}

We found that male Liolaemus lizards were consistently larger than females (Pincheira-Donoso \& Tregenza, 2011), across species (paired $t$-test, $t_{54}=6.692, p<0.001$; Figure S3). SSD showed credible evidence of phylogenetic signal, but having controlled for this, mean SSD was credibly male-biased among species $(99.3 \%$ of posterior samples of mean SSD were $>0$ ). This indicates that the initial source of SSD is linked to sexual identity, either via direct sexual selection or via a predisposition for males to evolve large (or females to evolve small) body size.

\section{Environmental predictors of species-richness and SSD}

345 Our analyses of candidate environmental drivers using model-averaged regressions of SSD against 
347 latitude (Figure 4a). Similar analyses of species-richness against environmental predictors revealed the only 348 statistically important predictor to be annual precipitation: species-richness increased with increasing rainfall 349 (Figure 4b). Structural equation modelling confirmed the minimal adequate set of environmental predictors (SSD influenced by latitude and vegetation; species richness influenced by rainfall; Table 1), and furthermore revealed that the negative correlation between SSD and species richness remains significant following control of environmental influences. Rival models, in which SSD predicted variation in species richness, or vice versa, were not supported in our SEMs (Table 1). Overall, the bivariate correlation between SSD and species richness remains significant, but each response is mediated by different environmental predictors.

\section{Discussion}

Our study provides a large-scale test of the niche-packing equivalence theory, at assemblage level and ecological timescales, that dimorphic sexes and species are rival units of niche-saturation during adaptive radiations and community assembly (Slatkin, 1984; Bolnick \& Doebeli, 2003; Butler et al., 2007). As predicted, our analyses reveal a negative relationship between the species-richness of lizard assemblages and the magnitude of SSD in their component species. Additionally, although we observed that the breadth of the body size distribution per species is constrained by species-richness, the evidence for the opposite pattern of speciesrichness constraining the size distribution breadth of either sex alone is non-significant, raising the possibility that there is an asymmetry in the effect of these variables on one another. Finally, our variance decomposition analyses confirm that Liolaemus assemblages are organized non-randomly such that body size variation is constrained to be partitioned into the "between-sexes" and "among-species" components: increases in one component are accompanied by decreases in the other.

bioclimatic variation across assemblages. Species-richness increases with increasing precipitation across

371 the diet of Liolaemus species (greater dietary diversity facilitates higher numbers of coexisting species given the 372 greater niche space). In contrast, while SSD is not influenced by rainfall, it increases with decreasing vegetation 373 complexity and with increasing southerly latitude. Therefore, SSD seems to be favoured in low-complexity, low 374 productivity environments. The latitudinal cline, however, remains unexplained. Our combined findings reveal a scenario consistent with a relationship between SSD and species-richness mediated by the environmental 
conditions that influence variation in available niche space per assemblage. However, despite these effects from agents of natural selection, it remains possible that sexual dimorphism has been influenced by sexual selection (see Andersson, 1994). Niche packing occurs against the backdrop of sexual selection on size dimorphism, with the potential for interactions between the two (for instance, where factors such as population density and species richness impact both ecological competition for resources and inter-male competition for mates). The action of sexual selection during the evolutionary histories of Liolaemus species presumably predisposes them to evolve dimorphism in the direction of males being larger than females, but our results suggest that this size difference is constrained in species-rich assemblages. We note that sexual dimorphism is greatest in habitats with low cover or complexity of vegetation, and suggest that this could be due to the importance of sexual signaling in simple habitats where visibility makes selection on display traits more intense, or due to more intense competition for scarce food resources.

Our core questions were (1) whether ecologically distinct sexes and species can saturate niches in equivalent ways, and hence, (2) whether such equivalence triggers the predicted conflict between sexual dimorphism and species-richness (Bolnick \& Doebeli, 2003; Butler et al., 2007). Our results reveal patterns consistent with the prediction that sexual dimorphism and species-richness are antagonistic. However, environments where resource availability is higher sustain more species, while highly dimorphic species are found in environments of low vegetation cover or complexity. Overall, despite strong evidence for a negative correlation between SSD and species-richness, we conclude that this tension is context-specific in Liolaemus. Our surveys of sexual dimorphism and species richness did not allow us to determine cause and effect, i.e., whether the two sources of body size variation compete equally for niche space during the processes of adaptive radiation and community assembly. We suggest that variation among species is more likely to constrain the evolution of sexual dimorphism, than vice versa, for two main reasons. First, sexual dimorphism is evolutionarily more labile than species formation (i.e., it evolves faster, requires simpler conditions, is reversible), perhaps because it requires only a direct effect of selection on ecological traits, rather than an additional indirect effect on mating behaviour (Bolnick \& Doebeli, 2003; Cooper et al., 2011). Second, the magnitude of ecological divergence between the sexes is typically small compared to the magnitude of ecological divergence among coexisting species. Hence, we argue that whenever niche space has not been saturated by other species, sexual dimorphism of ecologically relevant phenotypes may evolve by natural selection through its benefits for sex-specific fitness. Such benefits may include, for example, reduced intensity of resource competition between 
405 the sexes (Shine, 1989; Bolnick \& Doebeli, 2003), and relaxation of intralocus sexual conflict when intrinsic sex406 specific fitness-linked roles need to evolve in different directions under the same natural selection regimes 407 (Hedrick \& Temeles, 1989; Bonduriansky \& Chenoweth, 2009). Any attempt to tease apart the influence of 408 species richness on the evolution of sexual dimorphism and/or the colonisation of dimorphic species, from the 409 influence of sexual dimorphism on speciation and/or the colonisation of new species, would require either 410 massive-scale experimentation, or long-term observation of evolutionary and ecological event sequences. Our results suggest interesting nuances to the relationship between sexual dimorphism and adaptive 412 radiation. De Lisle \& Rowe (2015) show that sexual dimorphism is associated with diversification rate and 413 reduces extinction, hence promoting biodiversity on macroevolutionary timescales. Consequently, such positive 414 impacts on radiation would be compromised when the pressures of interspecific competition prevent divergence 415 between the sexes. The niche-packing equivalence theory raises novel possibilities to understand eco416 evolutionary dynamics by incorporating the role of intraspecific diversification into the traditionally species417 centred views of biodiversity evolution and community assembly.

\section{ACKNOWLEDGMENTS}

420 The authors thank David Hosken, Tim Barraclough, Trine Bilde, Lilly Harvey, Shai Meiri, Dave Shuker, and 421 Jonathan Chase for valuable insights on earlier versions of this manuscript. Alejandro Scolaro provided 422 extensive data and expertise on Patagonian assemblages of lizards. Three referees and Adriana Ruggiero made excellent critical comments to improve this paper. This study was funded by the Leverhulme Trust, and received additional support from the University of Lincoln through a RIF Grant to DPD. DJH was supported by NERC standard grant NE/L007770/1 and by NERC International Opportunities Fund NE/N006798/1.

\section{Biosketch}

428 Daniel Pincheira-Donoso is a Senior Lecturer in Evolutionary Biology. His research investigates the role of 429 selection as a driver of adaptive diversity, with a primary focus on the interplay between the emergence of 430 adaptive traits and their impact on large-scale patterns of diversity. 
433 The data supporting the results will be archived in an appropriate public repository such as Dryad or Figshare 434 and the data DOI will be included at the end of the article.

435

436

\section{REFERENCES}

Andersson, M. (1994) Sexual selection. Princeton University Press, Princeton.

Barton, K. (2017) MuMIn: Multi-model inference. R package version 1.40.0. R Foundation for Statistical Computing, Vienna.

Bolnick, D.I. \& Doebeli, M. (2003) Sexual dimorphism and adaptive speciation: two sides of the same ecological coin. Evolution, 57, 2433-2449.

Bonduriansky, R. \& Chenoweth, S.F. (2009) Intralocus sexual conflict. Trends in Ecology and Evolution, 24, 280288.

Brown, R.P., Znari, M., El Mouden, E.L.H. \& Harris, P. (1999) Estimating asymptotic body size and testing geographic variation in Agama impalearis. Ecography, 22, 277-283.

Butler, M.A., Sawyer, S.A. \& Losos, J.B. (2007) Sexual dimorphism and adaptive radiation in Anolis lizards. Nature, 447, 202-205.

Cei, J.M. (1986) Reptiles del centro, centro-oeste y sur de la Argentina. Herpetofauna de las zonas áridas y semiáridas. Museo Regionale di Scienze Naturali di Torino, Torino.

Cooper, I.A., Gilman, R.T. \& Boughman, J.W. (2011) Sexual dimorphism and speciation on two ecological coins: patterns from nature and theoretical predictions. Evolution, 65, 2553-2571.

Costa, G.C., Nogueira, C., Machado, R.B. \& Colli, G.R. (2007) Squamate richness in the Brazilian Cerrado and its environmental-climatic associations. Diversity and Distributions, 13, 714-724.

De Lisle, S.P. \& Rowe, L. (2015) Independent evolution of the sexes promotes amphibian diversification. Proceedings of the Royal Society of London B, Biological Sciences, 282, 20142213.

Fairbairn, D.J. (2007) The enigma of sexual size dimorphism. Sex, size \& gender roles. Evolutionary studies of sexual size dimorphism (ed. by D.J. Fairbairn, W.U. Blanckenhorn and T. Szekely), pp. 1-10. Oxford University Press, Oxford.

Fairbairn, D.J., Blanckenhorn, W.U. \& Szekely, T. (2007) Sex, size \& gender roles. Evolutionary studies of sexual size dimorphism. Oxford University Press, Oxford. 
461 Fox, J., Nie, Z., Byrnes, J., Culbertson, M., DebRoy, S., Friendly, M., Goodrich, B., Jones, R.H., Kramer, A. \& 462 Monette, G. (2017) sem. Structural equation models. R package version 3.1-9. R Foundation for Statistical 463 Computing, Vienna.

464 Gavrilets, S. (2004) Fitness landscapes and the origin of species. Princeton University Press, Princeton. 465 Hedrick, A.V. \& Temeles, E.J. (1989) The evolution of sexual dimorphism in animals: hypotheses and tests. 466 Trends in Ecology \& Evolution, 4, 136-138.

467 Hijmans, R.J., Cameron, S.E., Parra, J.L., Jones, P.G. \& Jarvis, A. (2005) Very high resolution interpolated 468 climate surfaces for global land areas. International Journal of Climatology, 25, 1965-1978.

469 Imhoff, M.L., Bounoua, L., Ricketts, T., Loucks, C., Harriss, R. \& Lawrence, W.T. (2004) Global patterns in 470 human consumption of net primary production. Nature, $\mathbf{4 2 9}, \mathbf{8 7 0 - 8 7 3 .}$

471 Losos, J.B. (2009) Lizards in an evolutionary tree. Ecology and adaptive radiation of anoles. University of $472 \quad$ California Press, Berkeley.

473 Losos, J.B. (2010) Adaptive radiation, ecological opportunity, and evolutionary determinism. American Naturalist, $474 \quad 175,623-639$.

Losos, J.B., Butler, M. \& Schoener, T.W. (2003) Sexual dimorphism in body size and shape in relation to habitat use among species of Caribbean Anolis lizards. Lizard social behaviour (ed. by S.F. Fox, J.K. Mccoy and T.A. Baird), pp. 356-380. John Hopkins University Press, Baltimore and London.

Meiri, S. (2008) Evolution and ecology of lizard body sizes. Global Ecology and Biogeography, 17, $724-734$.

Meiri, S., Kadison, A.E., Novosolov, M., Pafilis, P., Foufopoulos, J., Itescu, Y., Raia, P. \& Pincheira-Donoso, D. (2014) The number of competitor species is unlinked to sexual dimorphism. Journal of Animal Ecology, 83, $1302-1312$.

Nosil, P. (2012) Ecological speciation. Oxford University Press, New York.

Novosolov, M., Rodda, G.H., Feldman, A., Kadison, A.E., Dor, R. \& Meiri, S. (2016) Power in numbers. The 485 evolutionary drivers of high population density in insular lizards. Global Ecology and Biogeography, 25, 8795.

Peterson, A.T., Soberon, J., Pearson, R.G., Anderson, R.P., Martínez-Meyer, E., Nakamura, M. \& Araújo, M.B. (2011) Ecological niches and geographic distributions. Princeton University Press, Princeton.

Pincheira-Donoso, D. (2011) Predictable variation of range-sizes across an extreme environmental gradient in a lizard adaptive radiation: evolutionary and ecological inferences. PLoS One, 6, e28942. 
490 Pincheira-Donoso, D. \& Núñez, H. (2005) Las especies chilenas del género Liolaemus. Taxonomía, sistemática 491 y evolución. Publicación Ocasional del Museo Nacional de Historia Natural de Chile, 59, 1-487.

492 Pincheira-Donoso, D. \& Tregenza, T. (2011) Fecundity selection and the evolution of reproductive output and 493 sex-specific body size in the Liolaemus lizard adaptive radiation. Evolutionary Biology, 38, 197-207.

494 Pincheira-Donoso, D. \& Meiri, S. (2013) An intercontinental analysis of climate-driven body size clines in reptiles: 495 no support for patterns, no signals of processes. Evolutionary Biology, 40, 562-578.

496 Pincheira-Donoso, D., Hodgson, D.J. \& Tregenza, T. (2008a) The evolution of body size under environmental 497 gradients in ectotherms: why should Bergmann's rule apply to lizards? BMC Evolutionary Biology, 8, 68. 498 Pincheira-Donoso, D., Scolaro, J.A. \& Sura, P. (2008b) A monographic catalogue on the systematics and 499 phylogeny of the South American iguanian lizard family Liolaemidae (Squamata, Iguania). Zootaxa, 1800, 1$500 \quad 85$.

501 Pincheira-Donoso, D., Harvey, L.P. \& Ruta, M. (2015) What defines an adaptive radiation? Macroevolutionary 502 diversification dynamics of an exceptionally species-rich continental lizard radiation. BMC Evolutionary 503 Biology, 15, 153.

504 Pincheira-Donoso, D., Hodgson, D.J., Stipala, J. \& Tregenza, T. (2009) A phylogenetic analysis of sex-specific 505 evolution of ecological morphology in Liolaemus lizards. Ecological Research, 24, 1223-1231.

506 Pincheira-Donoso, D., Tregenza, T., Witt, M.J. \& Hodgson, D.J. (2013a) The evolution of viviparity opens 507 opportunities for lizard radiation but drives it into a climatic cul-de-sac. Global Ecology and Biogeography, 22 $508 \quad 857-867$.

509 Pincheira-Donoso, D., Bauer, A.M., Meiri, S. \& Uetz, P. (2013b) Global taxonomic diversity of living reptiles. $510 \quad$ PLoS One, 8, e59741.

511 Pincheira-Donoso, D., Jara, M., Reaney, A., García-Roa, R., Saldarriaga-Córdoba, M. \& Hodgson, D.J. (2017) 512 Hypoxia and hypothermia as rival agents of selection driving the evolution of viviparity in lizards. Global 513 Ecology and Biogeography, 26, 1238-1246.

514 Pincheira-Donoso, D., Fox, S.F., Scolaro, J.A., Ibargüengoytía, N., Acosta, J.C., Corbalán, V., Medina, M., 515 Boretto, J., Villavicencio, H.J. \& Hodgson, D.J. (2011) Body size dimensions in lizard ecological and 516 evolutionary research: exploring the predictive power of mass estimation equations in two Liolaemidae 517 radiations. Herpetological Journal, 21, 35-42. 
518 Poe, S., Goheen, J.R. \& Hulebak, E.P. (2007) Convergent exaptation and adaptation in solitary island lizards.

519 Proceedings of the Royal Society of London, Biological Sciences, 274, 2231-2237.

520 Schluter, D. (2000) The ecology of adaptive radiation. Oxford University Press, Oxford.

521 Schoener, T.W. (1969) Size patterns in West Indian Anolis lizards: I. Size and species diversity. Systematic

$522 \quad$ Zoology, 18, 386-401.

523 Schoener, T.W. (1977) Competition and the niche. Biology of the Reptilia. Vol. 7. Ecology and behaviour A (ed.

524 by C. Gans and D.W. Tinkle), pp. 35-136. Academic Press, London.

525 Schulte, J.A., Losos, J.B., Cruz, F.B. \& Núñez, H. (2004) The relationship between morphology, escape 526 behaviour and microhabitat occupation in the lizard clade Liolaemus (Iguanidae: Tropidurinae: Liolaemini).

$527 \quad$ Journal of Evolutionary Biology, 17, 408-420.

528 Scolaro, J.A. (2005) Reptiles patagónicos sur. Una guía de campo. Editorial Universidad Nacional de la 529 Patagonia, Trelew.

530 Shine, R. (1989) Ecological causes for the evolution of sexual dimorphism: a review of the evidence. Quarterly 531 Review of Biology, 64, 419-464.

532 Slatkin, M. (1984) Ecological causes of sexual dimorphism. Evolution, 38, 622-630.

533 Smith, R.J. (1999) Statistics of sexual size dimorphism. Journal of Human Evolution, 36, 423-459.

534 Stamps, J.A. \& Andrews, R.M. (1992) Estimating asymptotic size using the largest individuals per sample.

$535 \quad$ Oecologia, 92, 503-512.

536 Stephens, P.R. \& Wiens, J.J. (2009) Evolution of sexual size dimorphisms in emydid turtles: ecological 537 dimorphism, Rensch's rule, and sympatric divergence. Evolution, 63, 910-925.

538

539

540

541

542

543

544

545

546 


\section{FIGURE LEGENDS}

548 Figure 1. The relationships between sexual size dimorphism (SSD) and species-richness, described using (A) 549 mean SSD per assemblage, or (B) per-species SSD. Correlation and mixed-model regression analyses reveal 550 significant negative correlations compared to null expectations formed by permuting species among 551 assemblages. Modelling with phylogenetic control yields posterior distributions of variance components and a 552 regression slope that reveal $(C)$ credible phylogenetic signal in the residuals of the regression of SSD against 553 species-richness, but (D) a credibly negative relationship between SSD and richness, despite phylogenetic 554 control.

Figure 2. The relationships between species-richness and the breadth (standard deviation) of the body size distributions, either (A) per assemblage or (B) per species. Breadths correlate negatively with increasing species-richness. The body size distribution breadths of $(C)$ males and (D) females do not correlate significantly with species-richness.

Figure 3. Deviation of each assemblage from the line of constraint that describes apportioning of all variation to between-sexes or among-species components. (A) Positions of assemblages in relation to the line constraining the proportion of variance absorbed by "sex" or "species".

(B) Conversion of these data into the distance along the line of constraint (now the x-axis) and perpendicular distance of each assemblage from this line (now the yaxis). (C) Comparison of the total squared perpendicular distances of the observed assemblages from the hypotenuse (vertical arrow), against null distributions of 10,000 permuted assemblages (white $=$ complete shuffle of species among assemblages; light grey = constrained shuffle of sex differences; mid-grey = constrained shuffle of species differences). (D) Comparison of the curvature of a nonlinear regression of perpendicular distances from the line of constraint (vertical arrow), against null distributions of 10,000 permuted assemblages (colours as in(C)). In (C) and (D), the observed distance or curvature lies far from the main body of the null distributions, rejecting the null hypothesis in each case.

573 Figure 4. Model-averaged effect sizes of environmental predictors of (A) sexual size dimorphism, and (B) species-richness. Mean effect sizes are presented, with 95\% confidence intervals. 